\title{
APUPA Expertentisch
}

\author{
Einigkeit über ein interdisziplinäres Vorgehen
}

Zum ersten Expertenstammtisch nach der Gründung der Österreichischen Dekubitus Präventionsgesellschaft (APUPA) Ende vergangenen Jahres kamen Mitte Mai in Salzburg elf Teilnehmer aus verschiedenen Bundesländern. Der interdisziplinären Zusammensetzung der APUPA entsprechend, kamen die Vertreter aus den verschiedensten Bereichen des $\mathrm{Ge}$ sundheitswesens: Medizin, intra- und extramurale Pflege, Ausbildung und Versicherungsträger. Dass die Aktivitäten der APUPA auch von den jeweiligen Dienstgebern Ernst genommen werden, zeigte sich in der Möglichkeit, den Mitarbeitern ausgenommen die Ärzte - die Teilnahme während der Dienstzeit zu ermöglichen.

\section{Themen und Ziele}

Der Themenkreis umfasste Dekubitusprävention, Hilfsmittellogistik, Wissensmanagement und Datenerfassung. Als Ziele definierte die Tagung unter Moderation von Zita Kis Dadara, MSc, der Präsidentin der APUPA, die Identifizierung und Zusammenführung von Experten, den Erfahrungsaustausch, die Analyse der Grundlagen der Dekubitusprävention, die Identifikation von Stärken und Verbesserungspotentialen und die Ausweitung der APUPA-Aktivitäten in Österreich. Die Einführungspräsentation, die einen Einblick in die bisherige Erfassung der österreichischen Situation innerhalb der Dekubitusprävention gab und internationale Erkenntnisse erörterte, bildete auch die Diskussionsgrundlage für die Tagung.

\section{Datenerfassung und Auswertung}

Datenerfassung und Datenerhebung erfolgen mittels Pflege- und medizinischer Anamnese durch unterschiedliche Vorgangsweisen. Während für die Pflegedokumentation eine gesetzliche Regelung vorliegt, ist die ärztliche Dokumentation gesetzlich nicht definiert. Dies führt zu unterschiedlichen Vorgehensweisen innerhalb einer Berufsgruppe. Die interdisziplinäre Kommunikation zum Thema Dekubitusprävention kann zwar in Österreich auf gute Insellösungen auf Stations- ebenen oder Organisationen verweisen, landesweit gibt es jedoch keine einheitliche Regelung. In der Diskussion zeigte sich, dass die oberste Führung in der eigenen Organisation oder Einheit die Verantwortung für eine professionelle Vorgangsweise der Dekubitusprävention und ihrer Dokumentation tragen muss. Auch ist es die Aufgabe einer Organisation, eine zeitgerechte Dokumentation zu ermöglichen und auch einzufordern. Der Hinweis auf die Berufspflichten sei demnach nicht ausreichend, waren sich die Experten einig. Angeregt wurde, dass die APUPA eine Klassifikation der Dekubiti und eine Skala zur Risikoeinschätzung vorgibt. Die APUPA übernimmt die international anerkannte EPUAP Klassifikation, dies wird bereits mit der Freischaltung der Homepage bekannt gegeben. Die Einschätzungsskala wurde von der APUPA dagegen noch nicht definiert. International ist die Braden Skala das meistverwendete Einschätzungsinstrument. Internationale Aktivitäten sind dann möglich, wenn die Braden Skala auch einheitlich in Österreich verwendet wird.

\section{Wissensmanagement}

In der Ausbildung zur Gesundheitsund Krankenpflege stellt Dekubitusprävention einen festen Bestandteil dar, in der ärztlichen Ausbildung bzw. Fort - und Weiterbildung liegt die Situation differenzierter. Dekubitusprävention nimmt als Ausbildungsinhalt in der Gesundheits- und Krankenpflege acht Unterrichtseinheiten ein, wobei das Thema Dekubitusprävention auch in mehreren Dimensionen immer wieder einfließt. Die teilnehmenden Ärzte berichteten, dass die Dekubitusprävention nicht explizit in ihrer Ausbildung eingebunden ist.

Fort- und Weiterbildungen haben nur organisationsspezifisch einen höheren Stellenwert gewonnen. Das bedeutet auch, dass in vielen Krankenhäusern und in der Altenpflege das Thema Dekubitusprävention noch sehr ausbaufähig ist.

Die Teilnehmer weisen eindeutig auf einen Fort- und Weiterbildungsbedarf in der Pflege und Medizin hin. Die Definition

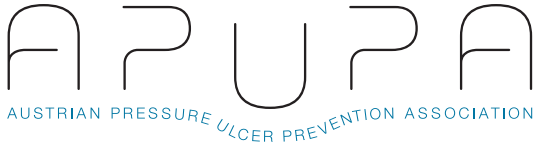

der Mindestanforderung der Wissenskomponenten zum Thema Dekubitusprävention ist demnach dringend notwendig und das Wissen sollte vom mittleren Management eingefordert werden. Das QM Instrument Wissensmanagement ist als Führungsinstrument $\mathrm{zu}$ sehen, denn die Einforderung der Umsetzung des erworbenen Wissens ist eindeutig Managementaufgabe. Die Teilnehmer wünschen die Nennung einer Expertengruppe seitens der APUPA, die zur Wissensvermittlung zur Verfügung stehen soll. Eine Informationsbroschüre über Dekubitus für die Betroffenen und deren Angehörige könnte erstellt werden, um Wissensdefizite zu verringern und konkrete Hilfestellungen zu geben.

\section{Hilfsmittellogistik}

Am Markt vorhandene zahlreiche Hilfsmittel und die Komplexität der Dekubitusprävention machen ein Hilfsmittelmanagement notwendig, betonten die Teilnehmer und beschlossen Maßnahmen zur Hilfestellung über die am Markt vorhandenen Hilfsmittel. Einige Experten äußerten ausdrücklich den Wunsch nach einer Projektgruppe innerhalb der APUPA für diese Aktivität. Daher werden drei Teilnehmer die Aktivitäten aufnehmen und einen Projektplan an die APUPA vorlegen. Die finanziellen Ressourcen für ein derartiges Projekt sind allerdings derzeit noch unsicher.

\section{Fazit}

Die Aktivitäten der APUPA zeigten sich nach der Tagung als sinnvoll, notwendig und werden fortgesetzt. Der Stellenwert derDekubitusprävention erhält derzeit nicht die notwendige Gewichtung und die professionelle Vorgehensweise muss unterstützt werden. Der interdisziplinäre Ansatz für die Aufarbeitung des Themas Dekubitusprävention in Österreich sei dringend notwendig, bestätigten die Experten.

Die Homepage der APUPA ist seit kurzem freigeschalten: www.apupa.at 